\title{
Global Well-Posedness to the three-dimensional Boussinesq system with nonlinear damping
}

\author{
Ridha SELMI ${ }^{1}$ and Mounia Zaabi ${ }^{2}$ \\ ${ }^{1} \mathrm{FSG}$ \\ ${ }^{2}$ Universite de Tunis El Manar
}

May 5, 2020

\begin{abstract}
In this paper, we prove the well-posedness of the solution to the three-dimensional Boussinesq system with nonlinear damping term. Existence, uniqueness and continuous dependence with respect to initial data of the weak solution are proved, under minimum regularity requirement in Sobolev and Lebegue spaces. Energy method, compactness method, and appropriate technical lemmas are used.
\end{abstract}

\section{Hosted file}

Ridha_Mounia_Sub_MMAS_19_02_2020.pdf available at https://authorea.com/users/300165/articles/ 429856-global-well-posedness-to-the-three-dimensional-boussinesq-system-with-nonlineardamping 